\title{
Denkmuster für die angewandte Geographie
}

\section{Einleitung}

Gemäß dem Titel dieses Symposiums geht es bei dieser Veranstaltung um unseren Lebensraum. Diese Wahl ist für eine Geographische Gesellschaft auch nicht so überraschend. Der Titel präzisiert jedoch, indem von der «Zukunft» unseres Lebensraumes die Rede sein soll. Dies ist schon eher überraschend. Ein 100-Jahr-Jubiläum könnte doch ebensogut zurückblenden auf die bisherige Entwicklung und auf die Sternstunden geographisch-ethnographischen Arbeitens. Wenn der Blick nun aber vorwärtsgerichtet ist, heißt das nicht, daß die bisherigen Leistungen minder geachtet würden. Im Gegenteil, wir Heutigen müssen uns sehr bemühen, die hohe Qualität des Denkens und Arbeitens unserer geistigen Väter halten zu können.

Ist von Lebensraum die Rede, gilt es, eine gewisse Präzisierung vorzunehmen. Wir wollen Lebensraum auf zwei Ebenen betrachten: Einerseits unseren eigenen engeren Lebensraum (d.h. unsere Wohn- und Arbeitsumgebung, unsere Städte und Landschaften, den Lebensraum der Schweiz usw.) andererseits sind dies nur Teile eines größeren Lebensraums. Dieses größere Ganze ist der Lebensraum unserer Erde insgesamt. Die beiden Ebenen sind miteinander verwoben.

Wie steht es um den Lebensraum Erde heute? Dieser Frage müssen wir deshalb kurz nachgehen, weil das Arbeiten auf lokaler Ebene getragen werden muß vom Blick auf das Ganze. Blättert man in neuerer Literatur, sind Darstellungen des Zustandes unseres Lebensraumes zu finden, die gewissermaßen von einer Weltuntergangsstimmung getragen werden. Ich zitiere aus SIEBKER (1983): «Nicht nur hat unser Zivilisationsspiel uns die Welt vergessen lassen, es hat sie auch so weit zerstört, daß unser eigener Spielraum eng geworden ist, die Tore zur Zukunft der Menschheit sich endgültig zu schließen drohen.» Siebker war Physiker und Mitglied des Club of Rome. 1980 übersiedelte er auf einen Gärtnerhof bei Wien und starb kurz darauf! Wie Siebker sind auch andere ausgestiegen und hoffen im Geiste Noahs die nahende Katastrophe zu überleben. Wenn wir gewisse urbane Lebensräume und gewisse Ereignisse betrachten, könnte tatsächlich Panik in einem aufkommen. Neulich, als ich diese Zeilen von Siebker las, war ich im Zug unterwegs zwischen
Oslo und Bergen. In meinem Tagebuch fand ich zu dieser fast $500 \mathrm{~km}$ langen Reise unter anderem: «Schnee, Granitfelsen, Wildwasser: sehr schön». Und auf der Rückreise: «Das Licht ist wesentlich besser geworden. Stellenweise scheint die Sonne. Die Landschaft ist noch viel schöner als vorher.» Von zerstörter Umwelt, von Weltuntergangsstimmung keine Spur!

Unweigerlich taucht die Frage auf, wieviel unsere Erde an menschlichem Wirken erträgt. Neulich kam mir ein Buch von LOVELOCK (1989) in die Hände. Darin legt er seine GAIA-Hypothese dar, die Hypothese, daß die Erde nicht ein physikalisches Gebilde, sondern ein lebendes Ganzes sei. Er weist unter anderem auf die Tatsache hin, daß trotz der Zunahme der Sonnenenergie-Einstrahlung um ein Viertel auf unseren Planeten die Temperatur auf der Erdoberfläche konstant geblieben ist.

Die Erde scheint demzufolge die Fähigkeit zu besitzen, «Störungen» soweit aufzufangen, daß die Bedingungen für die Erhaltung des Lebens nicht nachhaltig ändern. Wenn die Erde (als GAIA) mit den gewaltig höheren Sonneneinstrahlungen fertig wurde, wächst in einem die Zuversicht, daß sie auch mit unseren heute produzierten Umweltzerstörungen fertig werden könnte.

Die Herausforderung an uns besteht wohl darin, so zu handeln, daß die Grundpfeiler unseres Lebens auf der Erde (Erde, Wasser, Luft, Pflanzen) nicht so strapaziert werden, daß die großartige Selbstregulierung zusammenbrechen würde. Von diesem globalen Denken her muß unser Handeln im Lokalen bestimmt werden. An uns richtet sich die Herausforderung, unser Handeln auf die zu erwartenden Auswirkungen zu untersuchen, auf Kohärenz (bzw. Verträglichkeit) mit dem Ganzen zu beurteilen und die Konsequenzen bezüglich Tun und Lassen zu ziehen . Diese Ausführungen bilden gewissermaßen das Denkmuster auf der höheren Ebene. Steigen wir nun nach dieser Einführung auf der globalen Ebene wieder hinunter auf die Ebene unseres eigenen Wirkungskreises. Dabei will ich auf die folgenden Fragen eingehen: a) Welche Aufgaben haben wir zu lösen? b) Welches sind die Eigenheiten, die diese Aufgaben begleiten? c) Welches sind geeignete Denk-

Peter Gresch, Dr., PD, Lättenstraße 25, 8116 Würenlos 
muster, um vor diesem Hintergrund die gestellten Aufgaben anzugehen?

\section{Aufgaben, die zu lösen sind}

Aufgrund des vorher Gesagten interessiert uns also einerseits der Lebensraum und andererseits raumund umweltwirksames Handeln.

\section{Ausgangslage:}

Gegeben ist ein Ist-Zustand. Eine raum- und umweltwirksame Handlung wird realisiert. Es wird festgestellt, welche Wirkung die Handlung hat, das heißt, es wird untersucht, welche Veränderungen am Ist-Zustand bewirkt wurden (Abb. 2.1). Es ist bekannt, daß neben den aus der Sicht des Handelnden angestrebten positiven Wirkungen räumlich und umweltmäßig auch negative Wirkungen resultieren können. Durch die Enge des heutigen Lebensraumes ist es nicht mehr zulässig, aus sektorieller Sicht, ohne Rücksicht auf andere Interessen eine Maximal- beziehungsweise eine Optimallösung anzustreben. Bei großen Investitionen (zum Beispiel bei Infrastrukturanlagen) zeigt es sich auch, daß auftretende negative Wirkungen, wenn überhaupt, nur noch mit großen Aufwendungen sich korrigieren lassen.

\section{Aufgaben der Raum- und Umweltplanung:}

In der Raum- und Umweltplanung geht es unter anderem darum, bei (großen) Vorhaben die negativen Wirkungen (Probleme, Konflikte) gar nicht erst entstehen zu lassen. Auf der Basis des Vorsorgeprinzips verlangen sowohl das Raumplanungsgesetz als auch das Umweltschutzgesetz, daß vor der Realisierung eines raum- und umweltwirksamen Vorhabens die zu erwartenden Wirkungen aufgezeichnet werden (Wirkungsanalyse). Grundlage für diese Wirkungsanalyse sind einerseits die bestehende Situation (Ist-Zustand) und andererseits die Absicht zu einer Handlung.

- Eine 1. Aufgabe besteht darin, den Ist-Zustand hinreichend zu beschreiben.

- Die 2. Aufgabe besteht darin, mit Hilfe der Wirkungsanalyse die raum- und umweltmäßigen Auswirkungen auf die bestehende Situation zu beschreiben (Abb. 2.2).

- Mit der Beschreibung allein ist es jedoch noch nicht getan: Die zu erwartenden Wirkungen sind auch zu beurteilen, um die Zulässigkeit der Handlung abschätzen zu können. Die Basis der Beurteilung bilden die Vorstellungen über die erwünschte räumliche Entwicklung. Bewußt wurde der Begriff «Vorstellungen» in der Mehrzahl formuliert, denn

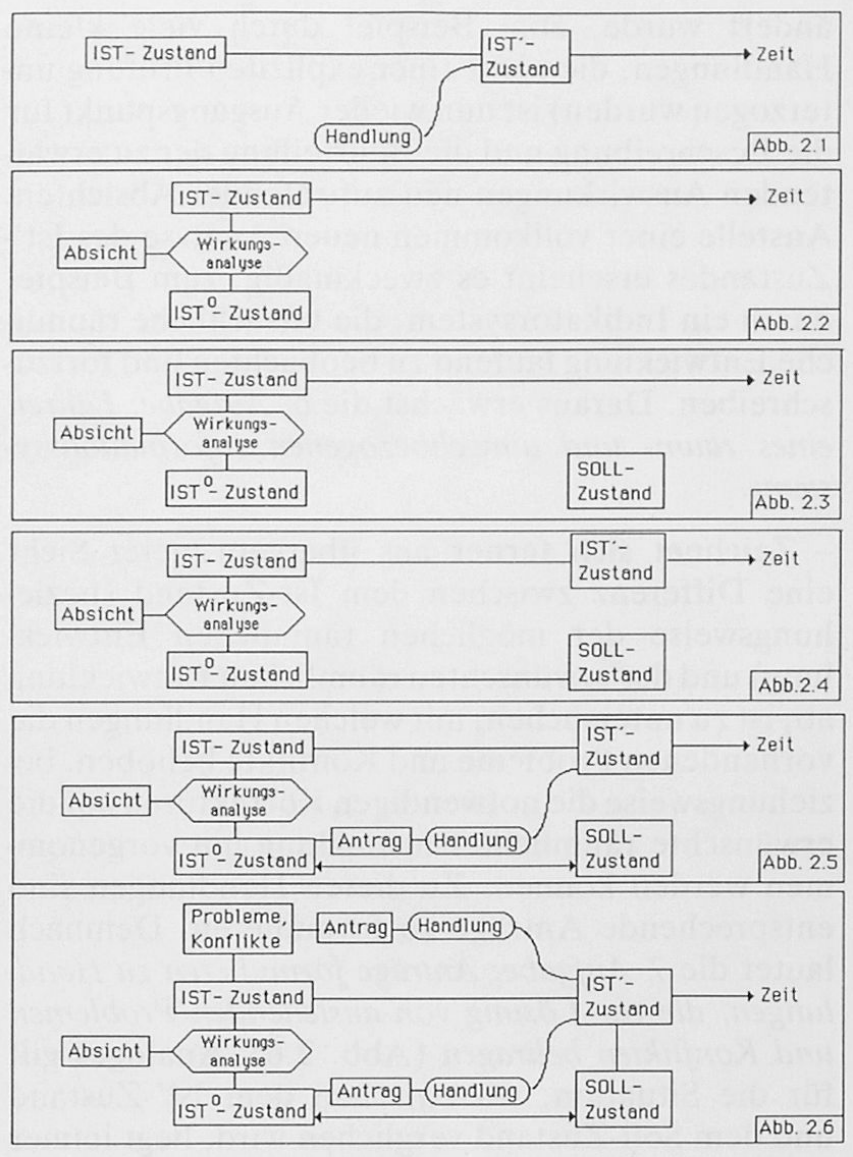

es gibt nicht die (eine) Vorstellung über die erwünschte räumliche Entwicklung. Die 3. Aufgabe lautet: Beschreiben erwünschter räumlicher Entwicklungen beziehungsweise Zustände. (Dabei wird von Überlegungen über mögliche räumliche Entwicklungen ausgegangen.) (Abb. 2.3)

- Nachdem die zu erwartenden Wirkungen beschrieben (Aufgabe 2) und der Soll-Zustand (Vorstellungen über erwünschte räumliche Entwicklung) definiert sind (Aufgabe 3), ist die Basis für die nächste Aufgabe gelegt: 4. Aufgabe: Beurteilen der zu erwartenden Auswirkungen der beabsichtigten Handlung vor dem Hintergrund des Soll-Zustandes (Abb. 2.4).

- Führt die beabsichtigte Handlung tatsächlich zu dem erwünschten Soll-Zustand, kann «grünes Licht» für die Realisierung der Handlung gegeben werden. Zeigt es sich jedoch, daß die beabsichtigte Handlung unter Abwägung der verschiedenen Interessen überwiegend negative Auswirkungen bringen würde, ist auf die Handlung zu verzichten. Die 5. Aufgabe besteht darin, einen Antrag auf Bewilligung (allenfalls mit Auflagen) beziehungsweise auf Ablehnung der Handlung zu formulieren. (Abb. 2.5)

- Nach der (allfälligen) Realisierung der Handlung liegt nun der ursprüngliche Ist-Zustand in modifizierter Form vor. Dieser neue Zustand (Ist') (der im übrigen durch andere Handlungen ebenfalls mitver- 
ändert wurde, zum Beispiel durch viele kleine Handlungen, die nicht einer expliziten Prüfung unterzogen wurden) ist nun wieder Ausgangspunkt für die Beschreibung und die Beurteilung der zu erwartenden Auswirkungen neu auftretender Absichten. Anstelle einer vollkommen neuen Analyse des Ist'Zustandes erscheint es zweckmäßig, zum Beispiel durch ein Indikatorsystem, die tatsächliche räumliche Entwicklung laufend zu beobachten und fortzuschreiben. Daraus erwächst die 6. Aufgabe: Führen eines raum- und umweltbezogenen Informationssystems.

- Zeichnet sich ferner aus übergeordneter Sicht eine Differenz zwischen dem Ist-Zustand (beziehungsweise der möglichen räumlichen Entwicklung) und der erwünschten räumlichen Entwicklung $a b$, ist zu untersuchen, mit welchen Handlungen die vorhandenen Probleme und Konflikte behoben, beziehungsweise die notwendigen Korrekturen auf die erwünschte räumliche Entwicklung hin vorgenommen werden können. $\mathrm{Zu}$ diesen Handlungen sind entsprechende Anträge zu formulieren. Demnach lautet die 7. Aufgabe: Anträge formulieren zu Handlungen, die zur Lösung von anstehenden Problemen und Konflikten beitragen (Abb. 2.6). Analoges gilt für die Situation, wo zwischen dem Ist'-Zustand und dem Soll-Zustand verglichen wird, liegt immer noch eine Differenz zwischen diesen beiden Zuständen vor (Probleme, Konflikte), sind ebenfalls Anträge zu deren Bereinigung zu formulieren.

\section{Zusammenfassung:}

Insgesamt ergibt sich aus dem zuvor Ausgeführten folgende Liste der zu lösenden Aufgaben:

Aufgabe 1: Beschreibung des Ist-Zustandes

Aufgabe 2: Beschreibung der zu erwartenden raumund umweltmäßigen Auswirkungen der beabsichtigten Handlung auf den IstZustand

Aufgabe 3: Beschreiben erwünschter räumlicher Zustände

Aufgabe 4: Beurteilen der zu erwartenden Auswirkungen der beabsichtigten Handlungen

Aufgabe 5: Formulieren von Anträgen zur Bewilligung bzw. Ablehnung von Handlungen

Aufgabe 6: Führen eines raum- und umweltbezogenen Informationssystems

Aufgabe 7: Formulieren von Anträgen zur Behebung von Problemen und Konflikten

\section{Eigenheiten des Umfeldes}

Betrachtet man die zu lösenden Aufgaben etwas genauer, fällt auf, daß sie durch eine ganze Menge von Eigenheiten geprägt sind, die auf die Wahl der Vorgehensweise einen nachhaltigen Einfluß haben. Ein
Teil der Eigenheiten hat ihren Ursprung in der Zeitachse, denn schwergewichtig befassen wir uns bei der Lösung obiger Aufgaben mit Zukünftigem. Damit verlassen wir den Boden des sicheren, gesicherten Wissens. Zweck dieser Ausführungen ist es herauszuschälen, ob wir es mit sogenannten gutstrukturierten oder mit schlechtstrukturierten Problemsituationen zu tun haben, denn je nachdem sind andere Denkmuster und Vorgehensweisen anzuwenden.

Charakteristische Eigenheiten des Umfeldes für die Lösung der Aufgaben:

\section{- Unsicherheit/Ungewissheit}

- Über Künftiges gibt es kein sicheres Wissen, und doch sind Überlegungen über mögliche künftige Entwicklungen anzustellen (bzw. Prognosen zu machen). Wir wissen demnach auch nie sicher, ob die von uns gestellten Anträge zu Handlungen ihre Wirkungen auch im erhofften Maß erfüllen. Wir handeln immer unter Risiko.

- Dynamik:

- Für die Beschreibung einer Situation läßt sich die Zeit nicht anhalten. Konstellationen im Raum sind einem steten Wandel ausgesetzt. Beurteilungskriterien können sich rasch ändern: Was gestern noch als wichtig angesehen wurde, ist morgen vielleicht nicht mehr so wichtig, usw.

\section{- Komplexität:}

- Wir haben es nicht mit geschlossenen, sondern mit offenen Systemen zu tun. Egal wo wir den Perimeter für die inhaltliche und geographische $\mathrm{Ab}$ grenzung legen, sind «Fremdeinflüsse» vorhanden. Das Umfeld wird immer einen Einfluß auf das zu untersuchende System haben.

- Es ist schwierig, die wichtigen Aspekte bzw. Elemente herauszufinden, und schwierig, die zwischen den Elementen bestehenden Wechselwirkungen zu erkennen.

- Wir haben es nicht mit einfachen linearen Beziehungen zu tun, sondern mit einem Netz von Beziehungen. Das einfache Ursachen-WirkungsSchema ist untauglich.

- Unvollständiges Wissen:

- Auch wenn wir uns noch so lange der Beschreibung einer Situation widmen, hätten wir nie vollständige Informationen.

\section{- Unscharfe Informationen:}

- Da wir uns vor der Realisierung eines Vorhabens befinden, haben wir es nicht mit feststellbaren Handlungen zu tun, sondern mit Absichten. Diese sind mitunter noch vage formuliert, und Auskünfte über die einzelnen Projektelemente liegen häufig noch gar nicht vor.

- Vielzahl von Beteiligten/Vielzahl von Zielen:

- Bei größeren Vorhaben ist immer eine Vielzahl 
von Beteiligten vorhanden, sei es im Umfeld des Auftraggebers oder des Auftragnehmers. Formell festgelegte Kompetenzen sind dabei vielfach weniger maßgebend als die effektive Einflußnahme der Beteiligten auf verschiedenen «Hierarchieebenen». Parallel zu dieser Vielzahl von Beteiligten gilt, daß eine Vielzahl von (z. T. recht unterschiedlichen) verschiedenen Zielvorstellungen darüber, was der Raum hergeben bzw. was die Umwelt ertagen soll, besteht. Die Vielzahl von Beteiligten bzw. von Zielen macht sich auch in den verschiedenen, für Raum und Umwelt relevanten Gesetzen bemerkbar, wo bisweilen widersprüchliche Bestimmungen festgelegt sind.

$\mathrm{Zu}$ diesen Charakteristiken kommt eine weitere, ebenso bedeutende Feststellung hinzu:

\section{- Situationen und Ereignisse sind in Raum und Zeit einmalig:}

- Wohl mag es ähnliche oder vergleichbare, nie aber gleiche Situationen geben. D.h. das, was einmal an einem Ort zweckmäßig war, ist es möglicherweise an einem anderen Ort gar nicht. Oder was einmal an einem Ort richtig war, muß es zu einem späteren Zeitpunkt nicht mehr sein.

Aus diesen Überlegungen lassen sich einige Erkenntnisse formulieren, die für die weitere Arbeit von Bedeutung sind:

- Wir haben es bei raum- und umweltrelevanten Fragestellungen selten (oder nie) mit sog. wohlstrukturierten, sondern mit schlechtstrukturierten Problemen zu tun.

- Im Gegensatz zur Lösung von wohlstrukturierten Problemen gibt es bei schlechtstrukturierten Problemen nie richtige Lösungen, sondern zulässige, wobei Zulässigkeit durch die Einhaltung gesetzter Randbedingungen gegeben wird.

- Die Denkmuster, Vorgehensweisen und Techniken, die wir von der Lösung wohlstrukturierter
Probleme her kennen, taugen für die Lösung von schlechtstrukturierten Problemen nichts oder wenig.

- Es sind andere Denkmuster und Vorgehensweisen zu wählen (heuristische Methoden), um die zuvor aufgelisteten Aufgaben meistern zu können.

- Erstaunlicherweise dominiert in der Geographie Literatur über die Lösung von wohlstrukturierten Problemen, während (ähnlich wie bei der Literatur über Operations Research) Einführungen in den Umgang mit schlecht strukturierten Problemen praktisch fehlen oder allenfalls, um diesem Bereich doch noch Rechnung zu tragen, kurz vor dem Schluß noch erwähnt werden. Mit den Ausführungen im nächsten Kapitel soll eine Einführung in zukunftsorientierte Denkmuster gegeben werden.

\section{Die Aufgaben der Fachleute für Raum- und Umweltfragen}

In den bisherigen Ausführungen wurde keine Differenzierung zwischen den verschiedenen Beteiligten vorgenommen. Zum besseren Rollenverständnis und als Vorbereitung auf spätere Ausführungen scheint es zweckmäßig, eine Klärung vorzunehmen. Grundsätzlich sind bei raum- und umweltwirksamen Vorhaben folgende Akteure beteiligt:

a) der Handelnde,

b) die Genehmigungs- bzw. Bewilligungsbehörde,

c) die Fachleute für Raum- und Umweltfragen.

a) und b) können als Auftraggeber auftreten, während die «Fachleute für Raum- und Umweltfragen» als Auftragnehmer zu sehen sind. Die Arbeit beginnt mit einem Auftrag und endet mit der Ablieferung der formulierten Anträge (GRESCH, 1988) (Abb. 4.1). Nach der Erteilung des Auftrages

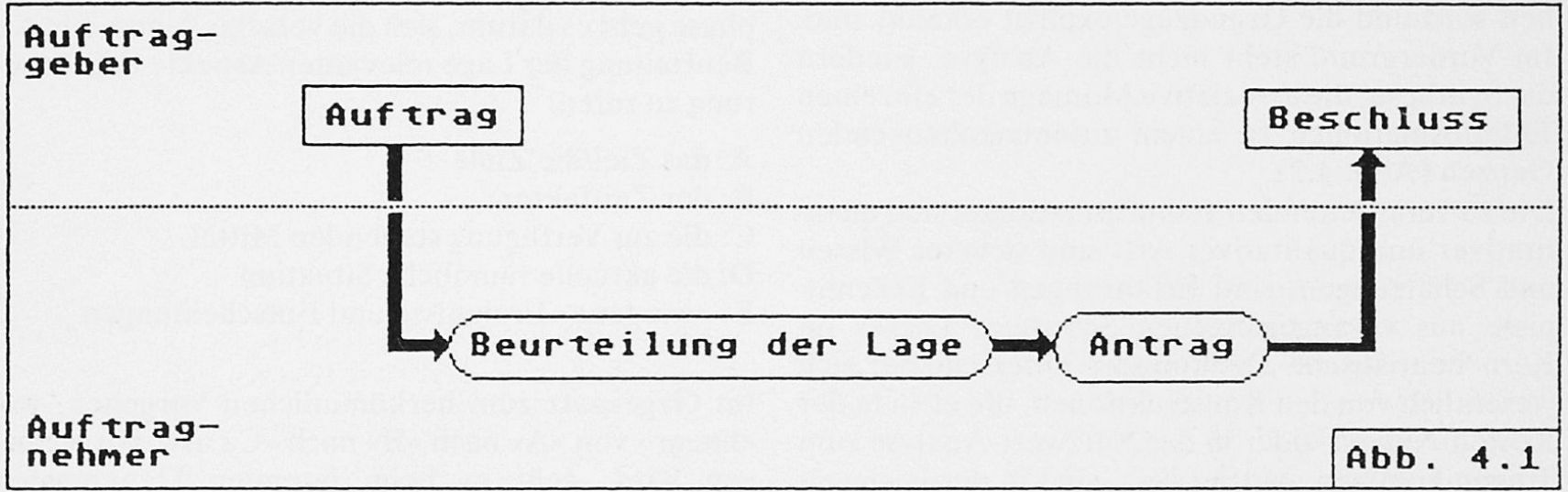


nimmt der Auftragnehmer eine Beurteilung der Lage vor, die ihn zum Entschluß über die Lösung der Aufgabe führt. Zuhanden des Auftraggebers formuliert der Auftragnehmer einen Antrag und begründet ihn. Die Grundlage für die Begründung kommt aus den Erkenntnissen im Rahmen der Lagebeurteilung. Dieses Bild ist insofern etwas vereinfacht, als ein Auftrag ja häufig nicht aus dem heiteren Himmel kommt und plötzlich da ist; voraus gehen etwa Gespräche und Offertstellung usw. Obwohl hier nicht besonders hervorgehoben, spielt diese erste Phase eine bedeutende Rolle, denn eine nicht sorgfältig ausgeführte Arbeit im Vorfeld der Auftragsformulierung kann zu großen Mißverständnissen und Ärger führen (z. B. wenn bei unklarem Auftrag Fragen beantwortet werden, die gar nicht gestellt sind, bzw. gestellte Fragen nicht beantwortet werden). Die Feststellung, daß die gröbsten Fehler jeweils ganz am Anfang gemacht werden, trifft hier ganz besonders zu. Eine zweckmäßige Lösung besteht meiner Meinung darin, daß der Experte bereits für die Formulierung des durch ihn zu erfüllenden Auftrags beigezogen wird. Das kann bei komplexen Aufgaben dazu führen, daß es in einer ersten Stufe darum geht, den zu bearbeitenden Auftrag zu formulieren. Kehren wir zurück zu dem durch den Auftragnehmer zu leistenden Teil der Arbeit, zur Beurteilung der Lage und zur Entschlußfassung.

Nachdem in der Einleitung ein Denkmuster auf der generellen (globalen) Ebene dargelegt wurde, geht es nun darum, auf der operationellen Ebene der Abwicklung konkreter Aufgaben und Aufträge ein entsprechendes Denkmuster zu entwickeln. Es geht also im folgenden darum, die Grundzüge eines Denkmusters zu entwerfen, das geeignet ist (sein soll), die gestellten Aufgaben zu lösen, und den dargelegten Eigenheiten (schlechtstrukturierte Probleme) Rechnung trägt. Der Einstieg in die Bearbeitung erfolgt nach der Erteilung des entsprechenden Auftrags durch den Auftraggeber. Entscheidend für eine erfolgreiche Beratertätigkeit ist die Fähigkeit, Anträge für Handlungen einfach formulieren und stichhaltig begründen zu können. Dies ist nur möglich, wenn auch komplexe, schlechtstrukturierte Probleme so weit aufgearbeitet werden können, daß die Übersicht über das Ganze gewonnen wird und die Grundzüge explizit erkannt sind. Im Vordergrund steht nicht die Analyse, sondern die Synthese, die assoziative Montage der einzelnen Teilinformationen zu einem zusammenhängenden Ganzen (Abb. 4.2).

Die zu verarbeitenden Teilinformationen sind quantitativer und qualitativer Art, sind sicheres Wissen und Schätzungen, sind Erfahrungen und Erkenntnisse aus vorangegangenen Schritten. Dieses im Kern heuristische Denkmuster unterscheidet sich wesentlich von den Konstruktionen, die etwa in der Kosten-Nutzen- oder in der Nutzwert-Analyse zum Tragen kommen. Mittlerweile sind in der Fachwelt

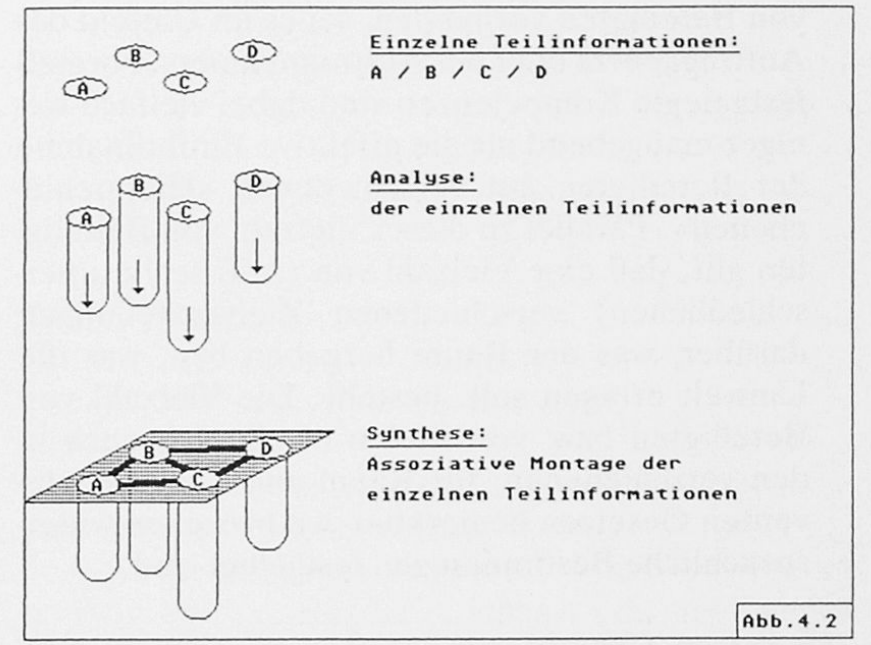

diesbezügliche algorithmische Vorgehensweisen auch ganz zu Recht in Verruf geraten. DE BONO (1985) hat (als Psychologe) mit seinem Ansatz des lateralen Denkens den für die Bearbeitung raumund umweltrelevanter Aufgaben zu begehenden Weg vorgezeichnet. Dadurch sind die Grundlagen für ganzheitliches, vernetztes Denken gelegt. ALLEN HICKLING und JOHN FRIEND (1987) basieren mit ihrem «strategic choice approach» letztlich auf demselben Denkmuster. Im wesentlichen geht es darum, durch sukzessive Annäherung an das Netz der relevanten Elemente, durch mehrmaliges Durcharbeiten der verschiedenen, zur Beurteilung der Lage gehörenden Fragenpakete den Entschluß zur Reife zu bringen.

Es ist hier nicht der Ort, um im Detail auf die Frage einzugehen, wie im einzelnen assoziative Montage, d. h. laterales Denken vor sich geht. Vielmehr sollen durch einige einführende Bemerkungen die Umrisse dieses Denkmusters illustriert werden. Im wesentlichen geht es bei dieser Montage um das Entwerfen von Handlungsmöglichkeiten und um das Herausarbeiten des Antrages.

Auf diesem Pfad sind verschiedene Phasen zu durchschreiten (Abb. 4.3). In einer Vorbereitungsphase geht es darum, sich die verschiedenen, für die Beurteilung der Lage relevanten Aspekte in Erinnerung zu rufen:
A: das Ziel/die Ziele
B: der Zeitfaktor
C: die zur Verfügung stehenden Mittel
D: die aktuelle räumliche Situation
E: «exogene» Ereignisse und Entscheidungen

Im Gegensatz zum herkömmlichen Vorgehen, wo «linear» von «A» nach «B» nach «C» usw. vorgegangen wird, geht es beim lateralen Denkmuster 
darum, zyklisch aus den verschiedenen Aspekten Informationen zusammenzutragen und zueinander in Beziehung zu bringen. Bei welchem Aspekt begonnen wird, spielt beim zyklischen Vorgehen keine Rolle. Die einzelnen Aspekte können im Rahmen dieses heuristischen Suchens mehr als einmal angegangen werden. Allmählich schälen sich im Rahmen der ersten Phase Handlungsmöglichkeiten heraus. Die generierten Lösungen gelten als zulässig, wenn die verschiedenen gesetzten Randbedingungen erfüllt sind.

Nach der Erstellung des Überblicks über die zulässigen Lösungen geht es in der zweiten Phase darum, die verschiedenen Lösungen gegeneinander abzuwägen, Vor- und Nachteile aufzulisten und in eine
Rangfolge zu bringen. Daraus resultiert der Entschluß.

In der dritten Phase geht es darum, den gefaßten Entschluss auf seine Stabilität hin zu prüfen: Führt die Handlung zu den gesetzten Zielen? Welche «exogenen» Entscheidungen und Entwicklungen können die vorgesehene Handlung in ihrer Wirkung schmälern? Es geht in dieser Prüfphase darum, die Stabilität des Entschlusses zu testen. Ist man überzeugt, über eine stabile Lösung zu verfügen, kann der Antrag zum Beschluß über die Handlung gestellt werden.

Ingesamt lassen sich alle sieben gestellten Aufgaben, die zuvor formuliert wurden, mit diesem Denkmuster der Beurteilung der Lage bearbeiten.

Aub. 4.3




\section{Folgerungen}

Um was ging es? Der Titel meines Referates lautete: «Denkmuster für die angewandte Geographie». Ich habe in meinen Ausführungen dargelegt, daß zwischen der Beschäftigung mit Vergangenem bzw. Gegenwärtigem und der Beschäftigung mit Zukünftigem (der Obertitel der Veranstaltung heißt ja: «Die Zukunft unseres Lebensraumes») grundsätzliche Unterschiede bestehen. Die Beschäftigung mit Zukünftigem verlangt, aufgrund der aufgezeichneten Eigenheiten bzw. Charakteristiken, nach andern, den Realitäten angepaßten Denkmustern als beim Arbeiten mit Vergangenem bzw. Gegenwärtigem. Im Vordergrund des Interesses steht dabei die Synthese (d. h. der Überblick über die Vernetzung) vieler Einzelinformationen. Diese Synthese ist jedoch nicht Selbstzweck, sondern geschieht - wenn wir von angewandter Geographie sprechen - mit Blick auf beabsichtigte Handlungen, das heißt mit Blick auf bevorstehende Entscheidungen. Das entworfene Denkmuster für die Beurteilung von Lagen mit zugehöriger Entschlußfassung wird getragen von den Grundzügen lateralen Denkens, wo heuristisch Assoziationen zwischen den relevant erscheinenden Elementen und Informationen hergestellt werden. Eine derartige Assoziation hatte z. B. Archimedes, als er in die Badewanne stieg und durch die Verdrängung von Wasser wesentliche Erkenntnisse über physikalische Gesetze gewann. Er soll dabei ausgerufen haben «Heureka - ich hab's». Das mit dem Fragenpaket zur Beurteilung der Lage aufgezeichnete Denkmuster soll helfen, systematisch auf den bezüglich einer Handlung zu formulierenden Antrag hinzuarbeiten. Die Herausforderung besteht sicher darin, aus der Fülle von Informationen, der Komplexität, der Ungewißheit und Unsicherheit Klarheit und Einfachheit zu erreichen. Ist dieses Denkmuster neuartig für die Geographie? Ich meine keineswegs, nur ist es im Laufe der zunehmenden Spezialisierungssucht auch innerhalb der Geographie verschüttet worden. Es fällt mir leicht, dies zu demonstrieren. Aus der Grundausbildung kennen wir das Modell der Geosphäre bestehend aus Lithosphäre, Atmosphäre, Hydrosphäre, Anthroposphäre (Abb. 5.1).

Im Geosphären-Modell sind die einzelnen Sphären aber nur ein Teil. Der andere Teil sind die Beziehun-

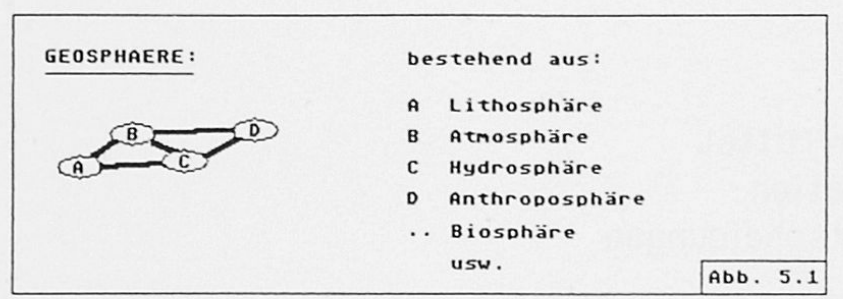

gen zwischen diesen Sphären, das Zusammenwirken oder, wie wir modern sagen würden, die Vernetzung zwischen diesen Elementen. Bilden wir nun die Assoziation zum lateralen Denken, ergibt sich nachfolgende und damit identische Darstellung (Abb. 5.2).

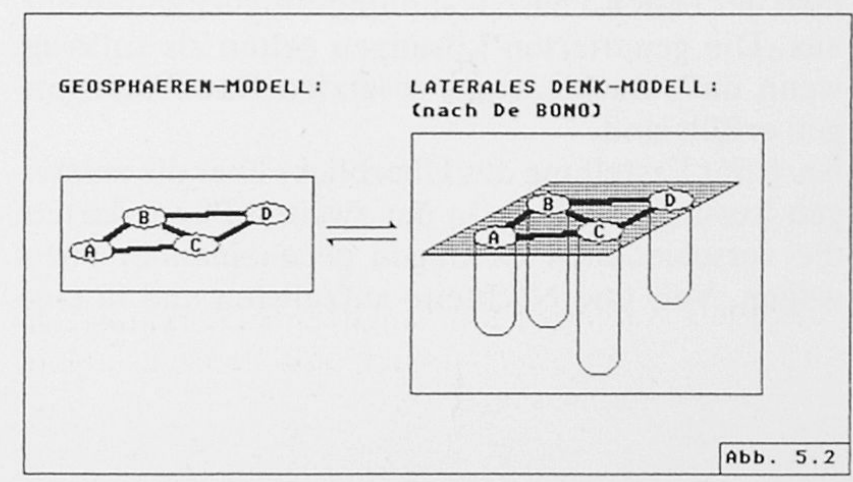

Betrachten wir die Geosphäre in diesem geradezu klassischen geographischen Sinne, stellen wir Kongruenz mit den heute eher gehörten Begriffen «Umwelt, Raum» fest. An obiger Darstellung kann im übrigen verdeutlicht werden, daß ein Vorstoß der Geographie in eine weitergehende Spezialisierung nicht der ursprünglichen Idee dient: Bei zu tiefem Eindringen geht die Kraft, um Querbeziehungen zu anderen Bereichen, Elementen zu sehen und zu verstehen, immer mehr verloren. Damit verliert der Geograph auch seine Qualifikation als Synthetiker. Will er seiner ureigenen Berufsbestimmung gerecht werden, hat er in die Analyse (vertikale Richtung) nur so weit vorzudringen, als er noch in der Lage ist, die Querbeziehungen zu den anderen Bereichen zu erkennen und zu verstehen. Er muß mit anderen Worten dialogfähig bleiben, sonst können keine Assoziationen, das Kernstück heuristischen Denkens, entstehen. Im übrigen ist festzustellen, daß sich bei den Leuten, die an der Nahtstelle zwischen vertikalem und lateralem Denken operieren, die Grenzen zwischen verschiedenen Disziplinen zunehmend verwischen. Einen «Synthetiker» stört das überhaupt nicht, im Gegenteil, er sucht ja das Gemeinsame.

Gestatten Sie mir noch eine Bemerkung zur neueren Entwicklung an den Hochschulen: Hier wird der Begriff «Umwelt» zunehmend als Werbeträger verwendet. Alte, disziplinär ausgelegte Vorlesungen in Chemie, Botanik, Zoologie, Ingenieurwissenschaften werden unter dem Titel Umweltlehre, Umweltnaturwissenschaft oder Umweltingenieurstudium verkauft: Alter Wein in neuen Schläuchen? ..., von lateralem Denken oder gar von heuristischem Annähern an Problemsituationen kann kaum oder eher keine Rede sein. Und die Geographie: Sie bedarf, wenn sie sich auf ihre ursprüngli- 
che Stärke besinnt, keines neuen Begriffs. Geographie ist auch in unserer Zeit ein hinreichend tragendes Fundament oder, um in der Bildsprache von vorher zu bleiben, geeignet, um «in alten Schläuchen neuen Wein» aufzunehmen.

Denken allein verwandelt die Welt nicht - nur Handlungen! Also, was tun? Ich sehe drei Adressatenkreise: a) wir als Individuen, b) wir als Mitglieder der Zürcherischen Geographisch-Ethnographischen Gesellschaft, c) wir als Lehrkörper an Hochschulen.

$\mathrm{Zu}$ a) was haben wir als Individuen (als Geographen) zu tun? Durch unsere Arbeiten erstens positiv einwirken auf die Erhaltung und Förderung der Qualität unseres Lebensraumes. Zweitens die anstehenden Aufgaben mit tauglichen Denkmustern anzugehen. Drittens erreichen, daß die Geographie in einer Art Renaissance wieder zurückfindet zu ihrer ursprünglichen Bestimmung als Disziplin der Synthese. Das Individuum vermag jedoch nicht alles. Es braucht die Gesellschaft, hier konkret die Geographisch-Ethnographische Gesellschaft Zürich.

$\mathrm{Zu}$ b) was haben wir als Vertreter dieser Gesellschaft zu tun? Ich sehe zwei Schwerpunkte: Erfahrungsaustausch, zum Beispiel dieses Symposium. Mit einer mittelfristigen Planung müßte hier auf der nationalen und internationalen Ebene etwas getan werden. Vielerorts ist Geographie zum Spezialistentum degeneriert. Allmählich wächst aber das Bewußtsein um die ursprüngliche Zweckbestimmung wieder. Die Gesellschaft sollte durch einen entsprechenden Erfahrungsaustausch auf nationaler und internationaler Ebene diese Kräfte wieder zusammenführen. Neben diesem Erfahrungsaustausch könnte die Gesellschaft gelegentlich auch Stellung beziehen zu aktuellen Zeitfragen, die unseren Lebensraum betreffen. In der bisweiligen Verwirrung der Bevölkerung durch Hiobsbotschaften sollte die Gesellschaft durch entsprechende Stellungnahme die Dinge wieder ins rechte Licht rücken.
Zu c) was haben wir als Lehrkörper an Hochschulen zu tun? Wäre es nicht sinnvoll, in der Grundausbildung von Geographen neben der Vermittlung von Wissen und Techniken - auch allenfalls unter Einbezug von Praktikern - ein Training in der Beurteilung von Lagen anzubieten? Wäre es nicht sinnvoll, durch das Anbieten von Weiterbildungsmöglichkeiten, die im Berufsleben stehenden Geographen periodisch im Bewältigen ihrer Aufgaben zu unterstützen und im geographischen Denken zu trainieren?

\section{Ich komme zum Schluß meiner Ausführungen}

Meinen Schlußsatz habe ich an der Wand der Garderobe des Geographischen Institutes der Universität Bristol gelesen. Dort war eingekritzelt: «When you are with a geographer - you are with someone spatial.» Dieses «spatial» war durchgestrichen und ersetzt mit «special».

\section{Literaturverzeichnis}

DE BONO, E. (1985): Lateral thinking. Penguin books, Harmondsworth (GB)

FRIEND, J., HICKLING A. (1987): Planning under pressure. Pergamon Press, Oxford

GRESCH, P. (1988): Raumplanerisches Denken. Unveröffentlichtes Manuskript zur Vorlesung an der ETHZ ws 88/89 Zürich

LOVELOCK, J. E. (1987): GAIA - A new Look at Life on Earth. Oxford University Press, Oxford

SIEBKER, M. (1983): Ökologie als Zentrale Zukunftsaufgabe in: KUNZ, G.: Die ökologische Wende. dtv, München 